\title{
Estudio de la movilidad de nanofluidos estables basados en hierro para remediación in situ de contaminantes inorgánicos
}

\section{Study of the mobility of stabilized iron based nanofluids for in situ remediation of inorganic pollutants}

Presentación: 6-7/10/2020

\section{Doctoranda:}

\section{Julieta Crespi}

Instituto Sabato, Comisión Nacional de Energía Atómica, Universidad Nacional de San Martín, Argentina

Centro de Tecnologías Químicas, Departamento de Ingeniería Química, Facultad Regional Buenos Aires, Universidad Tecnológica Nacional, Argentina

julicrespi@gmail.com

\section{Directora:}

\section{Natalia Quici}

\section{Co-director:}

\section{Víctor Nahuel Montesinos}

\section{Resumen}

Las nanopartículas de hierro cerovalente (FeNPs) se han empleado para la remoción de una gran variedad de contaminantes en medio acuoso, transformándolos en especies de menor toxicidad mediante mecanismos de adsorción superficial y reacciones rédox. En tratamientos de remediación in situ de aguas subsuperficiales se inyectan como nanofluidos (NFs), donde las suspensiones de FeNPs deben estabilizarse para evitar su precipitación y favorecer su transporte. En este trabajo se prepararon nanofluidos estables a partir de FeNPs empleando estabilizantes como carboximetilcelulosa (CMC) o goma xántica (GX), y se evaluó su movilidad en columnas rellenas de lecho poroso, a escalas de laboratorio y piloto. Los parámetros del lecho se obtuvieron por modelado de las curvas de ruptura de un trazador de $\mathrm{NaCl}$, empleando un software específico. Los NFs mostraron buena movilidad, mientras que las FeNPs sin estabilizar quedaron retenidas en el medio poroso. Con el objetivo de dilucidar completamente los mecanismos de transporte y los modelos de flujo actuantes se comenzó también con la caracterización reológica de los NFs, encontrándose resultados iniciales interesantes. Mediante una combinación adecuada de los experimentos de movilidad en columna, el modelado y la caracterización, se encontraron resultados alentadores que ayudarán a diseñar un sistema de inyección in situ de los NFs.

Palabras clave: Nanopartículas de hierro cerovalente, remediación subsuperficial, transporte.

\begin{abstract}
Zerovalent iron nanoparticles (FeNPs) have been used for the removal of a wide variety of contaminants in aqueous media, transforming them into less toxic species through surface adsorption and redox reactions mechanisms. For groundwater in situ
\end{abstract}


remediation treatments, FeNPs are injected as nanofluids (NFs), with the FeNPs forming stabilized suspensions that avoid their precipitation and to enhance their transport. In this work, stabilized suspensions of FeNPs were prepared using carboxymethylcellulose (CMC) or xanthic gum (GX), and their mobility was evaluated in porous bed columns, at laboratory and pilot scales. The bed parameters were obtained by modeling the breakthrough curves of a $\mathrm{NaCl}$ tracer, using a specific software. The NFs showed good mobility, while the not stabilized FeNPs remained retained in the porous media. To fully elucidate the transport mechanisms and the acting flow models, the rheological characterization of the NFs was also started, finding interesting initial results. Encouraging results were found through a combination of column mobility experiments, modeling and characterization, that will help to design an in situ injection system for NFs.

Keywords: Zerovalent iron nanoparticles, groundwater remediation, transport.

\section{Introducción}

Las nanopartículas de hierro cerovalente (FeNPs) se han empleado para la remediación de una gran variedad de contaminantes en medio acuoso. Son capaces de transformar a los contaminantes a través de adsorción superficial o mediante reacciones rédox en especies en solución menos tóxicas o más fácilmente eliminables (Zhang, 2003). En sistemas acuosos, se estudió con éxito la remoción de Cr(VI) (Montesinos et al., 2014), As(V) (Morgada et al., 2005) y U(VI) (Crespi et al., 2016; Quici et al., 2018), entre otros.

Para remediación de aguas subsuperficiales mediante tratamientos in situ, las nanopartículas (NPs) son inyectadas en el suelo como suspensiones, también llamadas nanofluidos (NFs). Una desventaja clave en estos tratamientos es la tendencia de las FeNPs a aglomerarse debido a fuerzas magnéticas y de Van der Waals (Phenrat et al., 2007). Así, la inyección se ve impedida o resulta ineficiente cuando las NPs forman agregados discretos de tamaños del orden de micrones o se agrupan en largas cadenas. Una posible solución ante esta problemática es el empleo de un revestimiento polielectrolítico, que inhiba la agregación de las NPs, mejorando así su estabilidad y transporte (Saleh et al., 2006). Por ejemplo, en años recientes se emplearon como recubrimiento carboximetilcelulosa (CMC) (Kocur et al., 2013), poliaspartato, sulfonato de poliestireno y ácido poliacrílico (Phenrat et al., 2008, Cirtiu et al., 2011). También se ha mostrado que la goma xántica (GX) es un estabilizante excelente, que mejora en gran medida la movilidad de las FeNPs (Comba y Sethi, 2009; Dalla Vecchia et al., 2009).

Por otra parte, un paso clave en el diseño de la inyección de los NFs implica estimar de forma confiable los parámetros operativos (distribución de partículas alrededor del pozo de inyección, el radio de influencia para una concentración objetivo, etc.) al desarrollar procedimientos y enfoques para evaluar la movilidad de las NPs en situaciones reales. El enfoque experimental requiere de la realización de experimentos de transporte en columnas y la determinación de la distribución de las NPs a la salida de la columna y en el interior de la misma en función del tiempo. La adición del uso de herramientas de simulación y modelado permite reducir la cantidad de ensayos necesarios para tales estimaciones. El programa Micro- and Nanoparticle transport, filtration and clogging Model Suite (MNMs 2018), desarrollado por el Politécnico de Torino, es una herramienta útil para modelar el transporte de NPs.

Finalmente, para completar el modelado de las ecuaciones de flujo y transporte de los NFs en suelo, es necesario conocer su comportamiento reológico ante variaciones de velocidad y presión que pueden darse al momento de la inyección, y para prever la movilidad subsuperficial de las NPs luego de inyectadas. Debido a lo expuesto anteriormente, los objetivos de este trabajo fueron, en primer lugar, estudiar el transporte de los NFs a dos escalas (laboratorio y piloto) para poder obtener datos experimentales plausibles de ser modelados empleando el software MNMs y, en segundo lugar, comenzar con la caracterización reológica de los NFs a fin de poder dilucidar completamente los mecanismos de transporte y los modelos de flujo actuantes.

\section{Desarrollo}


Se prepararon NFs empleando un protocolo de estabilización desarrollado previamente, basado en un procedimiento de estabilización post-síntesis, es decir, dispersando suspensiones de FeNPs comerciales en soluciones conteniendo los polímeros y polielectrolitos de interés.

Las FeNPs comerciales que se emplearon fueron NSTAR (Nanoiron s.r.o.), que presentan una estructura tipo core-shell, con núcleo de $\mathrm{Fe}(0)$ y capa externa de óxidos de hierro. Los estabilizantes elegidos fueron carboximetilcelulosa (CMC) y goma xántica (GX). Las soluciones de estabilizante se prepararon pesando la cantidad necesaria de material y llevando a volumen con agua Milli-Q (resistividad $=18 \mathrm{M} \Omega \mathrm{cm}^{-1}$ ) y agitando durante $1 \mathrm{~h}$ en un agitador orbital. Las soluciones obtenidas se mantuvieron refrigeradas $\left(\sim 4{ }^{\circ} \mathrm{C}\right)$ y cerradas herméticamente hasta su uso. Se prepararon suspensiones de NSTAR mediante pesada y adición de agua Milli-Q hasta alcanzar una concentración $\left[\mathrm{Fe}\left(\right.\right.$ total)] $=220 \mathrm{~g} \mathrm{~L}^{-1}$; la homogeneización de la suspensión se realizó empleando un homogeneizador portátil (Pro Scientific) durante 10 minutos. Luego, se realizó una dilución con agua de la suspensión original alcanzando $20 \mathrm{~g} \mathrm{~L}^{-1}$ de Fe(total) y se agitó por 20 minutos en una lavadora ultrasónica (Cleanson). A continuación, se preparó la suspensión final formada por una cantidad necesaria de suspensión de NSTAR $20 \mathrm{~g} \mathrm{~L}^{-1}$ de Fe(total) y de solución de estabilizante bajo agitación orbital durante 1 hora, para obtener las concentraciones finales deseadas de $\mathrm{Fe}$ (total) y estabilizante. Las suspensiones se nombraron de acuerdo con la siguiente nomenclatura: CMC $x-y$ NSTAR y GX $x-y$ NSTAR, donde $x$ corresponde a la concentración del estabilizante e $y$ corresponde a la concentración de NSTAR, respectivamente; ambas en g L1. En el caso de las suspensiones con $1 \mathrm{~g} \mathrm{~L}^{-1}$ de $\mathrm{Fe}$ (total), se omite el uso de $y$. Adicionalmente, se prepararon suspensiones de NSTAR sin estabilizar (b-NSTAR) como blanco de comparación, con la misma concentración de Fe(total), siguiendo el mismo procedimiento, pero sin agregar el estabilizante.

Para estudiar la movilidad de los NFs se realizaron ensayos a escalas laboratorio y piloto, empleando columnas rellenas de lecho poroso. Como lecho se empleó arena previamente tratada para eliminar toda contaminación posible. La columna se colocó verticalmente y el NF se hizo circular en sentido ascendente empleando una bomba peristáltica. La alimentación a la columna se hizo desde un reservorio conteniendo el NF bajo agitación mecánica constante empleando un agitador vertical. A la salida de la columna, se colectaron muestras a las que se les determinaron el $\mathrm{pH}$, la conductividad y la turbidez, esta última como medida de concentración de las FeNPs (empleando un turbidímetro portátil marca Hach). Luego de alcanzar una concentración estacionaria a la salida, la columna fue lavada con agua, estudiándose así la elución del material de hierro posiblemente retenido en la columna. Con los valores de turbidez obtenidos se graficaron las curvas de ruptura.

Para los ensayos realizados a escala laboratorio se emplearon columnas de vidrio de $1 \mathrm{~cm}$ de diámetro y $25 \mathrm{~cm}$ de largo, con filtros de lana de vidrio en la zona inferior y superior, y una altura de lecho de arena de $21 \mathrm{~cm}$. El caudal empleado fue de $12 \mathrm{~mL}$ $\mathrm{min}^{-1}$. Para los ensayos realizados a escala piloto, se empleó una columna de acrílico de $50 \mathrm{~cm}$ de largo y $4 \mathrm{~cm}$ de diámetro interno, con filtros de grava y lana de vidrio. En este caso, el caudal fue de $40 \mathrm{~mL} \min ^{-1}$ y la altura de lecho de $46 \mathrm{~cm}$.

Con el objetivo de evaluar el tiempo de retención característico de las columnas, se realizaron, en primer lugar, ensayos con una solución de $\mathrm{NaCl}$ como trazador. La concentración de $\mathrm{NaCl}$ a la salida de la columna fue determinada midiendo la conductividad de la solución con un conductímetro (Hach, SensION). Posteriormente, estas curvas se emplearon para modelar el lecho a ambas escalas empleando el software MNMs.

Si bien están pendientes los resultados del modelado de las curvas de los NFs, se comenzó una caracterización reológica de los mismos, con el fin de dilucidar los mecanismos de flujo que intervienen en el transporte de las FeNPs. Sobre los NFs se realizaron las determinaciones en un reómetro híbrido Discovery HR2, TA Instruments, del Centro de Tecnologías Químicas, Departamento de Ingeniería Química, Facultad Regional Buenos Aires, Universidad Tecnológica Nacional. La geometría empleada fue de plato y cono, de $2,0^{\circ}$ y $40 \mathrm{~mm}$ de diámetro. Se realizaron ensayos oscilatorios y de flujo.

\section{Resultados}


En primer lugar, se realizaron ensayos de transporte del trazador en ambas columnas. Las curvas de ruptura fueron modeladas satisfactoriamente empleando el programa MNMs, en ambos casos, obteniéndose ajustes con $\mathrm{R}^{2}$ superiores a 0,97. A partir de estos modelos, se encontraron para ambos lechos los valores de porosidad $(0,25$ a escala laboratorio y 0,22 a escala piloto) y dispersividad $(0,01 \mathrm{~m}$ a escala laboratorio y $0,004 \mathrm{~m}$ a escala piloto) correspondientes. Los valores obtenidos son semejantes entre sí, lo que implica que los lechos resultan similares respecto de estos parámetros, y podría esperarse que los lechos se comporten de manera equivalente respecto al tiempo de retención característico.

La Figura 1(a) muestra las curvas de ruptura de los experimentos realizados en la columna de laboratorio empleando tres NFs con $1 \mathrm{~g} \mathrm{~L}^{-1}$ de Fe(total): b-NSTAR, CMC10-NSTAR y GX5-NSTAR, comparadas con la curva del trazador. La Figura 1(b) muestra la curva de ruptura del experimento realizado en la columna a escala piloto con CMC5-0,5NSTAR, comparada con la curva del trazador.

A partir de lo mostrado en la Figura 1(a), puede observarse que, al estudiar el comportamiento de una suspensión sin estabilizante (b-NSTAR), las FeNPs quedan retenidas por completo en el lecho, acumulándose en la base de la columna, sin poder detectarse presencia de NPs en el flujo de salida. Cuando se alimentó la columna con agua con el objetivo de lavar el lecho y eluir las FeNPs retenidas, se obtuvieron muestras a la salida de la columna con concentraciones menores al $1 \%$ de la concentración de entrada (resultados no mostrados). Al finalizar el experimento, el 99,9\% del material permaneció retenido en la columna. Por el contrario, se obtuvo buena movilidad con ambos NFs estabilizados. Como se observa en la Figura 1(a), las NPs alcanzaron la salida de la columna en menos de 2 volúmenes de poro (VP), aunque con pendientes iniciales menores que la del trazador (siendo la pendiente del trazador el límite de máxima movilidad), debido a una mayor viscosidad de las suspensiones de NFs respecto a la solución de trazador (He y col., 2009). Para el caso de CMC10-NSTAR, se observa que la suspensión alcanza una concentración de NPs a la salida igual al $80 \%$ de la concentración de entrada para 8 VP y, luego, una caída de concentración que podría deberse a una obturación parcial de los poros del lecho (Tiraferri y Sethi, 2009). En el caso de GX5- NSTAR, se alcanzó la concentración de entrada (luego de $8 \mathrm{VP}$ ), lo que evidencia mayor movilidad que para CMC10-NSTAR. En los ensayos con ambos NFs, las NPs retenidas eluyeron por completo al alimentar la columna con agua en la etapa de lavado (resultados no mostrados).
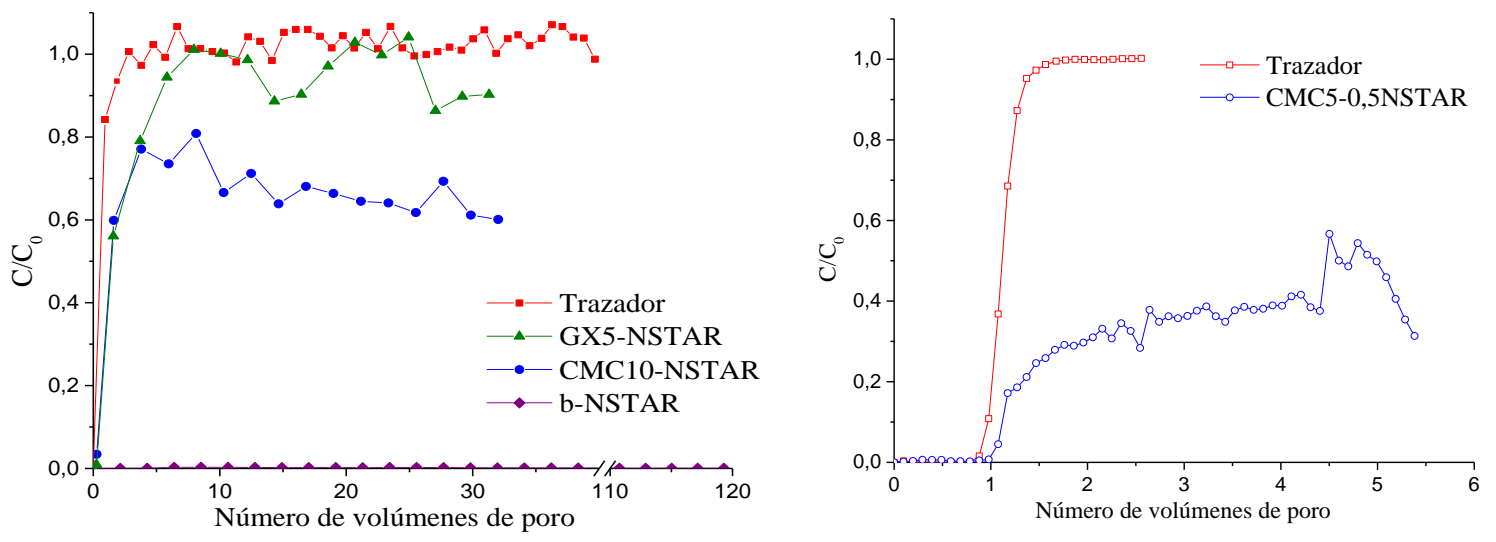

Figura 1: (a) Curvas de ruptura obtenidas a escala laboratorio con tres nanofluidos con $1 \mathrm{~g} \mathrm{~L}^{-1}$ de Fe(total): b-NSTAR, CMC10-NSTAR (10 g L-1 de CMC) y GX5-NSTAR (5 g L - $^{-1}$ de GX), comparadas con la del trazador. (b) Curvas de ruptura obtenidas a escala piloto con CMC5-0,5NSTAR (5 g $\mathrm{L}^{-1}$ de CMC y $0,5 \mathrm{~g} \mathrm{~L}^{-1}$ de Fe(total)) comparada con la del trazador.

Para los experimentos en planta piloto, se eligió emplear NFs preparados a partir de CMC debido a la disponibilidad y precio del estabilizante, dado que presentó resultados alentadores en los experimentos de laboratorio. Se decidió emplear una concentración de NPs menor, ya que se empleó un volumen mucho mayor de NF, conservando la relación CMC:FeNPs. Como muestra la Figura 1(b), se obtuvo buena movilidad con el NF, alcanzándose una concentración a la salida igual al 30\% de la 
concentración de entrada luego de $2 \mathrm{VP}$, y mayor al 50\% luego de $5 \mathrm{VP}$. Al lavar la columna, se comprobó que todo el material retenido previamente fue eluido de la columna (resultados no mostrados).

Si realizamos una comparación entre las curvas de NF con CMC en ambas escalas, mostradas en las Figuras 1(a) y 1(b), la tendencia pareciera opuesta: en la columna de laboratorio, la curva tiene un máximo y luego disminuye, mientras que la de planta piloto aumenta gradualmente. Sin embargo, la comparación no es directa, ya que la curva a escala de laboratorio es casi 20 veces mayor en número de volúmenes de poro que la obtenida en plata piloto. Queda pendiente un experimento a escala piloto con mayor volumen de NF para estudiar si la evolución de esta curva es similar a la obtenida en escala laboratorio. La diferencia en los máximos alcanzados podría ser explicada por el hecho de que una mayor velocidad favorece el arrastre de NPs (Raychoudhoury et al., 2014), siendo la velocidad de flujo a escala laboratorio 5 veces mayor que la empleada a escala piloto $\left(15,3 \mathrm{~cm} \mathrm{~min}{ }^{-1}\right.$ vs. $\left.3,18 \mathrm{~cm} \mathrm{~min}^{-1}\right)$. Además, el NF en los experimentos en laboratorio contenía el doble de concentración de CMC que el empleado en escala piloto, lo que implica una mayor viscosidad, lo que previene en mayor medida la sedimentación gravitacional (Tosco y Sethi, 2010).

Por otro lado, se comenzó con la caracterización reológica de soluciones de CMC $10 \mathrm{~g} \mathrm{~L}^{-1}$ y GX $5 \mathrm{~g} \mathrm{~L}^{-1}$, y de los NFs CMC10NSTAR y GX5-NSTAR. Si bien los resultados son parciales, se encontraron algunas conclusiones interesantes que colaborarán con el análisis de las ecuaciones de transporte.

A partir de los ensayos oscilatorios, se estudiaron las componentes elástica (G') y viscosa (G”) del módulo de corte y, en particular la relación entre ellos a partir del ángulo de fase $\delta$ (siendo tg $\delta=\mathrm{G}^{\prime \prime} / \mathrm{G}^{\prime}$ ). Tanto para la solución de CMC como para CMC10-NSTAR se obtuvieron módulos viscosos mayores a los elásticos, indicando un comportamiento más cercano de un líquido ideal $\left(\delta=90^{\circ}\right)$ que al de un sólido ideal $\left(\delta=0^{\circ}\right)$. A bajas frecuencias oscilación (hasta $\left.5 \mathrm{rad} \mathrm{s}^{-1}\right) \delta$ adquiere valores entre 80 y $85^{\circ}$, mostrando un alto componente viscoso, tendiendo casi al líquido ideal (con valores apenas mayores para CMC10NSTAR que para CMC). A frecuencias mayores (a partir de $60 \mathrm{rad} / \mathrm{s}$ ) estos valores disminuyen por debajo de $75^{\circ}$, e incluso por debajo de $70^{\circ}$ a $100 \mathrm{rad} / \mathrm{s}$, aumentando la importancia relativa de la componente elástica. Cuando se sometió a las muestras a un $100 \%$ de deformación oscilatoria, el ángulo de ambas muestras superó los $80^{\circ}$, y continuó aumentando hasta superar los $85^{\circ}$.

Para la solución de GX y para GX5-NSTAR los resultados fueron diferentes. A deformación y frecuencia de oscilación constantes ( $1 \%$ de deformación y $\left.6 \mathrm{rad} \mathrm{s}^{-1}\right)$, se encontró para ambas muestras un comportamiento mayormente elástico, con $\delta$ oscilando alrededor de los $27^{\circ}$. Sin embargo, para una deformación aplicada de $100 \%$ variando la frecuencia, se encontró que para GX $\delta$ se mantuvo por encima de los $48^{\circ}$ (aumentando levemente a altas frecuencias), mostrando un comportamiento apenas más viscoso que elástico, mientras que para GX5-NSTAR se mantuvo entre 35 y 40․ Finalmente, al aumentar la deformación (a frecuencia constante) se encontró que $\delta$ continuó aumentando para ambas muestras, alcanzándose casi los $75^{\circ}$ para GX5 y $70^{\circ}$ con GX5-NSTAR. El punto de cruce de ambos módulos (donde el ángulo es igual a los $45^{\circ}$ ) se alcanzó con 90\% de deformación para GX5 y $178 \%$ para GX5-NSTAR.

El análisis de los resultados de los ensayos de flujo aún está incompleto, pero hasta el momento pudo confirmarse que todas las muestras se comportan como fluidos no newtonianos, pseudoplásticos. Las muestras CMC y CMC10-NSTAR presentaron comportamientos similares, con una variación en la viscosidad del orden de 0,01 Pa s. Adicionalmente, ambas muestras presentaron una pequeña tixotropía (16 $\mathrm{Pa} \mathrm{s}^{-1}$ para CMC10 y $9 \mathrm{~Pa} \mathrm{~s}^{-1}$ para CMC10-NSTAR), que deberá ser estudiada con mayor profundidad. Para las muestras GX y GX5-NSTAR también el comportamiento resultó similar, con una pequeña variación en la viscosidad (de casi 0,02 Pa s para alta velocidad de deformación), sin embargo, presentaron una gran diferencia en la tixotropía, pasando de un comportamiento de tixotropía negativa en GX5 (-85 $\left.\mathrm{Pa} \mathrm{s}^{-1}\right)$ a una tixotropía casi nula pero positiva para GX5NSTAR $\left(1 \mathrm{~Pa} \mathrm{~s}^{-1}\right)$. Resulta necesario continuar con estos ensayos, probablemente variando la carga de NPs en el estabilizante para analizar cómo influyen estas en el comportamiento reológico del material.

\section{Conclusiones}


En este trabajo fue posible estudiar la movilidad los NFs estabilizados en lechos porosos a dos escalas distintas. En primer lugar, se verificó mediante modelado computacional que ambas escalas resultaron comparables a partir de los parámetros de lecho encontrados. Ambos estabilizantes estudiados (CMC y GX) mejoraron en gran medida la movilidad de las FeNPs. Las curvas obtenidas en ambas escalas deberán ser modeladas empleando el software MNMs para dilucidar el mecanismo de transporte actuante. Por otro lado, fue posible comenzar con la caracterización reológica de los NFs obteniéndose información relevante que deberá ser analizada en mayor detalle para complementar los modelos matemáticos que se obtengan a partir de los ensayos de movilidad. Mediante una combinación adecuada de los experimentos de movilidad en columna, el modelado y la caracterización, se encontraron resultados alentadores que ayudarán a diseñar un sistema de inyección in situ de los NFs.

\section{Referencias}

Cirtiu, C. M., Raychoudhury, T., Ghoshal, S., Moores, A. (2011). Systematic comparison of the size, surface characteristics and colloidal stability of zero valent iron nanoparticles pre- and post-grafted with common polymers. Colloids Surfaces A Physicochem. Eng. Asp., 390, 95. https://doi.org/10.1016/j.colsurfa.2011.09.011

Comba, S., Sethi, R. (2009). Stabilization of highly concentrated suspensions of iron nanoparticles using shear-thinning gels of xanthan gum. Water Res., 43, 3717. https://doi.org/10.1016/j.watres.2009.05.046

Crespi, J., Quici, N., Halac, E. B., Leyva, A. G., Ramos, C. P., Mizrahi, M., Requejo, F. G., Litter, M. I. (2016). Removal of Uranium (VI) with Iron Nanoparticles. Chem. Eng. Trans., 47, 265. https://doi.org/10.3303/CET1647045

Dalla Vecchia, E., Luna, M., Sethi, R. (2009). Transport in porous media of highly concentrated iron micro-and nanoparticles in the presence of xanthan gum. Environ. Sci. Technol., 43, 8942. https://doi.org/10.1021/es901897d

He, F., Zhang, M., Qian, T., Zhao, D. (2009). Transport of carboxymethyl cellulose stabilized iron nanoparticles in porous media: Column experiments and modeling. J. Colloid Interface Sci., 334, 96. https://doi.org/10.1016/j.jcis.2009.02.058

Kocur, C. M., O’Carroll, D. M., Sleep, B. E. (2013). Impact of nZVI stability on mobility in porous media. J. Contam. Hydrol., 145, 17. https://doi.org/10.1016/j.jconhyd.2012.11.001

MNMs 2018, https://areeweb.polito.it/ricerca/groundwater/software/mnms/.

Montesinos, V.N., Quici, N., Halac, E. B., Leyva, A. G., Custo, G., Bengio, S., Zampieri, G., Litter, M. I. (2014). Highly efficient removal of $\mathrm{Cr}(\mathrm{VI})$ from water with nanoparticulated zerovalent iron : Understanding the $\mathrm{Fe}(\mathrm{III})-\mathrm{Cr}(\mathrm{III})$ passive outer layer structure. Chem. Eng. J., 244, 569. https://doi.org/10.1016/j.cej.2014.01.093

Morgada, M. E., Levy, I. K., Salomone, V., Farías, S. S., López, G., Litter, M. I. (2009). Arsenic (V) removal with nanoparticulate zerovalent iron: Effect of UV light and humic acids. Catal. Today, 143, 261. https://doi.org/10.1016/j.cattod.2008.09.038

Phenrat, T., Saleh, N., Sirk, K., Tilton, R. D., Lowry, G. V. (2007) Aggregation and Sedimentation of Aqueous Nanoscale Zerovalent Iron Dispersions. Environ. Sci. Technol. 41, 284. https://doi.org/10.1021/es061349a

Phenrat, T., Saleh, N., Sirk, K., Kim, H.-J., Tilton, R. D., Lowry, G. V. (2008). Stabilization of aqueous nanoscale zerovalent iron dispersions by anionic polyelectrolytes: Adsorbed anionic polyelectrolyte layer properties and their effect on aggregation and sedimentation. J. Nanoparticle Res., 10, 795. https://doi.org/10.1007/s11051-007-9315-6

Quici, N., Meichtry, M., Montesinos, V. N. (2019). Use of Nanoparticulated Iron Materials for Chromium, Arsenic, and Uranium Removal from Water. En: Iron Nanomaterials for Water and Soil Treatment. Pan Stanford Publishing, Singapore, 177.

Raychoudhury, T., Tufenkji, N., Ghoshal, S. (2014). Straining of polyelectrolyte-stabilized nanoscale zero valent iron particles during transport through granular porous media. Water Res., 50, 80. https://doi.org/10.1016/j.watres.2013.11.038

Saleh, N., Sirk, K., Liu, Y., Phenrat, T., Dufour, B., Matyjaszewski, K., Tilton, R. D., Lowry, G. V. (2006). Surface Modifications Enhance Nanoiron Transport and NAPL Targeting in Saturated Porous Media. Environ. Eng. Sci., $24,45$. https://doi.org/10.1089/ees.2007.24.45

Tiraferri, A., Sethi, R. (2009). Enhanced transport of zerovalent iron nanoparticles in saturated porous media by guar gum. J. Nanoparticle Res., 11, 635. https://doi.org/10.1007/s11051-008-9405-0

Tosco, T., Sethi, R. (2010). Transport of Non-Newtonian Suspensions of Highly Concentrated Micro- And Nanoscale Iron Particles in Porous Media: A Modeling Approach. Environ. Sci. Technol., 44, 9062. https://doi.org/10.1021/es100868n

Zhang, W.-X. (2003). Nanoscale iron particles for environmental remediation- An overview. J. Nanoparticle Res., 5, 323. https://doi.org/10.1023/A:1025520116015 